\title{
USO DE TRATAMENTO HIDROTÉRMICO E ÁCIDO CLORÍDRICO NA QUALIDADE DE LICHIA 'BENGAL'
}

\author{
ELLEN TOEWS DOLL HOJO², JOSÉ FERNANDO DURIGAN², RONALDO HISSAYUKI HOJO², \\ JULIANA RODRIGUES DONADON², RAMILO NOGUEIRA MARTINS ${ }^{2}$
}

RESUMO - Um dos maiores problemas na pós-colheita da lichia é o escurecimento do pericarpo, cuja cor vermelha se torna totalmente escurecida em 48 horas, sob $25{ }^{\circ} \mathrm{C}$. Tecnologias que possam controlar o escurecimento do pericarpo são valiosas, e é o foco principal da pesquisa na área de pós-colheita. O objetivo deste trabalho foi avaliar a vida útil de lichias 'Bengal', armazenadas a $20^{\circ} \mathrm{C}$ e $82 \%$ UR após o tratamento hidrotérmico e/ou imersão em solução de $\mathrm{HCl}$. O delineamento experimental foi o inteiramente casualizado, em esquema fatorial $4 \times$ 7, com 3 repetições, onde o primeiro fator correspondeu aos tratamentos: testemunha; imersão em $\mathrm{HCl}$ a $1 \%$, por 6 minutos; tratamento hidrotérmico, com imersão em água a $52^{\circ} \mathrm{C}$ por 1 minuto, seguido de resfriamento em água a $10^{\circ} \mathrm{C}$, por 6 minutos; e tratamento hidrotérmico, seguido de resfriamento em $\mathrm{HCl}$ a $1 \%$, a $10^{\circ} \mathrm{C}$, por 6 minutos. O segundo fator foram os períodos de armazenamento: $0 ; 1 ; 2 ; 3 ; 6$; 9 e 12 dias. A combinação entre tratamento hidrotérmico $\left(52^{\circ} \mathrm{C}\right.$ por 1 minuto $)$ e resfriamento em $\mathrm{HCl}$ a $1 \%$ permite conservar a coloração das lichias 'Bengal' por até dois dias. Mesmo assim há escurecimento em $25 \%$ da superfície.

Termos para indexação: Litchi chinensis, escurecimento do pericarpo, antocianina, peroxidase, polifenoloxidase.

\section{USE OF HYDROTHERMAL AND ACID TREATMENT IN THE QUALITY OF 'BENGAL' LITCHI}

\begin{abstract}
One of the biggest problems in postharvest litchi is the pericarp browning, the color red becomes totally dark in 48 hours at $25^{\circ} \mathrm{C}$. Technologies that can control the browning of the pericarp are valuable, and it is the main focus of research in the post-harvest. The aim of this study was to evaluate the shelf life of 'Bengal' litchi, stored at $20^{\circ} \mathrm{C}$ and $82 \%$ RH after the hydrothermal treatment and/or immersion in $\mathrm{HCl}$ solution. The experimental design was completely randomized in a $4 \mathrm{x} 7$, with three replications, where the first factor was the treatment: control, immersion in $\mathrm{HCl} \mathrm{1 \%}$, for 6 minutes; hydrothermal treatment, soaking in water at $52^{\circ} \mathrm{C}$ for 1 minutes, followed by immersion in water at $10^{\circ} \mathrm{C}$ for 6 minutes, and hydrothermal treatment and subsequent cooling in $1 \% \mathrm{HCl}, 10^{\circ} \mathrm{C}$ for 6 minutes. The second factor was the storage periods: $0,1,2,3,6,9$ and 12 days. The combination of hydrothermal treatment $\left(52^{\circ} \mathrm{C}\right.$ for 1 $\mathrm{min}$ ) and cooling in $\mathrm{HCl} 1 \%$ allowed retaining the color of 'Bengal' litchi for up to two days. Yet there are browning in $25 \%$ of the surface.
\end{abstract}

Index terms: Litchi chinensis, pericarp browning, anthocyanins, peroxidase, polyphenol oxidase.

\section{INTRODUÇÃO}

Um dos maiores problemas da lichia, na sua pós-colheita, é o escurecimento do pericarpo, cuja cor vermelha se torna totalmente escurecida em 48 horas, sob $25^{\circ} \mathrm{C}$. Este escurecimento tem sido parcialmente atribuído à degradação da antocianina, por enzimas oxidativas, tais como a polifenoloxidase (PPO), peroxidase (POD) e ácido ascórbico oxidase (UNDERHILL, 1992). Este escurecimento também pode estar relacionado com o ataque de patógenos, dessecação do pericarpo e outros fatores ainda não conhecidos, como a colheita em épocas com temperaturas mais altas e o aumento do $\mathrm{pH}$ da seiva da casca (HOLCROFT; MITCHAM, 1996). Relata-se, também, que o escurecimento da casca é devido ao colapso celular, o que permite o contato das enzimas com seus substratos, resultando em oxidação dos substratos na presença de oxigênio e a produção de substâncias com coloração escura (LICHTER et al.,

\footnotetext{
(Trabalho 092-10). Recebido em: 26-04-2010. Aceito para publicaão em: 19-01-2011. Parte de tese de Doutorado em Agronomia do primeiro autor, apresentado ao Departamento de Produção Vegetal - FCAV -UNESP Jaboticabal.

${ }^{2}$ Engenheiros Agrônomo, doutorandos em Produção Vegetal da FCAV/UNESP - Jaboticabal. Via de Acesso Prof. Paulo Donato Castellane, s/n, 14884-900, Jaboticabal - SP. E-mail: ellendollhojo@yahoo.com.br; ronaldo.hojo@yahoo.com.br; julianadonadon@yahoo.com.br; ramilomartins@yahoo.com.br

${ }^{3}$ Professor Titular do Departamento de Tecnologia FCAV/UNESP - Jaboticabal. E-mail: jfduri@fcav.unesp.br
} 
2000). Por esta razão, retardar ou reduzir a oxidação enzimática é um importante método para aumentar o período de armazenamento e preservar a qualidade comercial de lichias.

A fumigação com dióxido de enxofre tem sido o tratamento mais usado para prevenir este escurecimento (UNDERHILL et al., 1997), mas tem sido evitado, pois deixa resíduos indesejáveis, altera o sabor do fruto e resulta em riscos à saúde dos consumidores e aos trabalhadores das casas de embalagens. A Europa, Austrália e Japão permitem um resíduo máximo de 10 ppm e, nos Estados Unidos da América, ele só está registrado para uso na pós-colheita de uva.

Um tratamento alternativo ao enxofre é o uso de ácidos, dado seu efeito na estabilidade da cor de antocianinas e na atividade de oxidases. Dentre eles, o ácido clorídrico é o mais usado, principalmente em associação com fumigação por enxofre (ZAUBERMAN et al., 1991; JIANG et al., 2004). Outra técnica utilizada é a aplicação de calor por tratamento hidrotérmico (LICHTER et al., 2000), que apresenta como principais vantagens a relativa facilidade de utilização, tratamento em um curto espaço de tempo, livre de resíduos, reduzindo assim a atividade da polifenoloxidase e também a concentração de $\mathrm{SO}_{2}$ aplicada sobre os frutos.

Outros tratamentos também têm sido sugeridos para reduzir este escurecimento, principalmente com o uso de ácido ascórbico ou cítrico, quitosana, lecitina, ceras e acondicionamento em embalagens plásticas (JIANG; FU, 1999; SIVAKUMAR; KORSTEN, 2006).

O objetivo deste trabalho foi avaliar a vida útil de lichias 'Bengal', armazenadas a $20^{\circ} \mathrm{C}$ e 82 \%UR após o tratamento hidrotérmico e/ou imersão em solução de $\mathrm{HCl}$.

\section{MATERIAL E MÉTODOS}

Utilizaram-se frutos de lichia (Litchi chinensis Sonn.) variedade Bengal, colhidos maduros, casca completamente avermelhada. Depois de préselecionados, foram acondicionados em caixas plásticas forradas com plástico-bolha e cuidadosamente transportados até o Laboratório de Tecnologia dos Produtos Agrícolas do Departamento de Tecnologia, da FCAV-UNESP, em até 2 horas. Os frutos foram selecionados e padronizados quanto ao tamanho, cor e ausência de injúrias, antes de receberem o tratamento de desinfestação, ou seja, imersão por 5 minutos em água clorada (200 mg. $\mathrm{L}^{-1}$ de hipoclorito de sódio), e deixados escorrer por 3 minutos.

Três amostras, com 7 frutos cada, foram avaliadas, antes da aplicação dos tratamentos, a fim de caracterizar os frutos no dia da colheita. Os frutos apresentaram peso médio de $13,9 \pm 0,7 \mathrm{~g}$; sólidos

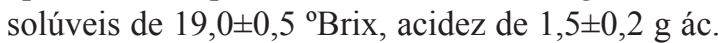
málico $100 \mathrm{~g}^{-1}, 57,7 \pm 6,9 \mathrm{mg} 100 \mathrm{~g}^{-1}$ de ácido ascórbico e $\mathrm{pH}=3,2 \pm 0,2 ; \quad$ aparência $=5,0 ; \quad 63,9 \pm 8,0 \mathrm{mg}$ $100 \mathrm{~g}^{-1}$ de antocianina e atividade de 4,5 $\pm 1,1 \mu \mathrm{mol}$ de fenol $\mathrm{min}^{-1} \mathrm{~g}^{-1}$ para a polifenoloxidase e $4,4 \pm 0,8$ nmol de $\mathrm{H}_{2} \mathrm{O}_{2} \min ^{-1} \mathrm{~g}^{-1}$ para a peroxidase.

Utilizou-se delineamento estatístico inteiramente casualizado, disposto em esquema fatorial $4 \mathrm{x}$ 7 , com 3 repetições, onde o primeiro fator correspondeu aos tratamentos: Testemunha (sem tratamento); Imersão em $\mathrm{HCl}$ a $1 \%$, por 6 minutos; Tratamento hidrotérmico, por imersão em água a $52{ }^{\circ} \mathrm{C}$ por 1 minuto, seguido de resfriamento em água a $10{ }^{\circ} \mathrm{C}$, por 6 minutos; e Tratamento hidrotérmico, seguido de resfriamento em $\mathrm{HCl}$ a $1 \%$, a $10^{\circ} \mathrm{C}$, por 6 minutos. $\mathrm{O}$ segundo fator foram os períodos de armazenamento: $0 ; 1 ; 2 ; 3 ; 6 ; 9$ e 12 dias. Cada parcela foi composta por 7 frutos, mantidos em embalagem rígida de poliestireno $(2,5 \mathrm{~cm} \times 22,4 \mathrm{~cm} \times 14,8 \mathrm{~cm})$, que foram armazenadas a $20{ }^{\circ} \mathrm{C} \pm 0,7{ }^{\circ} \mathrm{C}$ e $82 \%$ UR

As amostras foram analisadas quanto aos seguintes parâmetros: perda de massa fresca (\%), calculada pela diferença entre a massa inicial dos frutos e a obtida em cada tempo da amostragem, utilizando-se de balança semianalítica; aparência das frutas, que foi avaliada visualmente, usando-se uma escala de notas, em que , $5=$ vermelho-brilhante; $4=25 \%$ da casca escurecida; $3=50 \%$ da casca escurecida; $2=75 \%$ da casca escurecida; e $1=$ totalmente escurecida. Na polpa, foram determinados os teores de sólidos solúveis ( ${ }^{\circ} \mathrm{Brix}$ ), usando-se refratômetro digital ATAGO PR-100 (AOAC, 1997, proc. 920.151); acidez titulável ( $\mathrm{g}$ de ácido málico $100 \mathrm{~g}^{-1}$ ), por titulação com $\mathrm{NaOH}$ a $0,1 \mathrm{M}$, tendo como indicador fenolftaleína (AOAC, 1997, proc. 932-12); ácido ascórbico (mg $\left.100 \mathrm{~g}^{-1}\right)$, usando-se 2,6 diclorofenolindofenol de sódio a $0,1 \%$ para titular o extrato obtido da polpa com ácido oxálico a $0,5 \%$, a $5{ }^{\circ} \mathrm{C}$ (RANGANNA, 1977); SS/AT, calculada pela relação entre os teores de sólidos solúveis e de acidez titulável. Na casca, determinou-se o teor de antocianina ( $\mathrm{mg} 100 \mathrm{~g}^{-1}$ ), utilizando-se do método que tem como extrator uma mistura de etanol a $95 \%$ e $\mathrm{HCl}$ a $1,5 \mathrm{M}$ (15:85, v:v) e determinação colorimétrica (FRANCIS, 1982). A atividade da peroxidase ( $\mu$ mol de $\mathrm{H}_{2} \mathrm{O}_{2}$ consumido $\left.\mathrm{min}^{-1} \mathrm{~g}^{-1}\right)$ e da polifenoloxidase $(\mu \mathrm{mol} \mathrm{de}$ fenol consumido $\mathrm{min}^{-1} \mathrm{~g}^{-1}$ ) foi determinada na casca e na polpa dos frutos, utilizando-se de sobrenadante de amostras homogeneizadas em tampão fosfato de potássio a $0,2 \mathrm{M}, \mathrm{pH} 6,7$, e centrifugadas a $11655 \mathrm{xg}$, por 10 minutos, a $4{ }^{\circ} \mathrm{C}$. A atividade da peroxidase 
foi determinada pelo método de Allain et al. (1974), com utilização de $\mathrm{H}_{2} \mathrm{O}_{2}$ como substrato e leitura espectrofotométrica a $505 \mathrm{~nm}$, e a da polifenoloxidase pelo mesmo método, com utilização de fenol como substrato e leitura a $420 \mathrm{~nm}$. A atividade respiratória dos frutos, expressa em $\mathrm{mL}$ de $\mathrm{CO}_{2} \mathrm{~kg}^{-1} \mathrm{~h}^{-1}$, foi determinada em cromatógrafo Finningan, modelo 9001 (Finningan Corporation, San Jose, EUA), equipado com coluna de aço inox preenchida com Porapack-N e peneira molecular $(5 \mathrm{~A})$, detectores de condutividade térmica $\left(150^{\circ} \mathrm{C}\right)$ e de ionização de chama $\left(150^{\circ} \mathrm{C}\right)$, e que usa nitrogênio como gás de arraste $\left(30 \mathrm{~mL} \mathrm{~min}^{-1}\right)$. A amostra contendo 7 frutos foi mantida em frasco hermético $(2.000 \mathrm{~mL})$ por 80 minutos. Amostras com $0,3 \mathrm{~mL}$ da atmosfera deste ambiente foram tomadas no início e após o tempo de contenção no ambiente hermético. Os teores de $\mathrm{O}_{2}$ e $\mathrm{CO}_{2}$ foram calculados usando-se o "software" Borwin (Borwin version 1.20, JMBS Developpements, Le Fontanil, França).

Os dados obtidos foram submetidos à análise de variância, e a descrição das variáveis, em função dos períodos de armazenamento, foi feita utilizandose da análise de regressão, e os modelos foram selecionados observando a significância do teste $\mathrm{F}$ para cada modelo e seus respectivos coeficientes de determinação.

\section{RESULTADOS E DISCUSSÃO}

A perda de massa aumentou linearmente durante o período de armazenamento, independentemente dos tratamentos, de 7,0\%, em 1 dia, para 28,6\%, após 12 dias (Figura 1A). Maiores perdas de massa significam também maior escurecimento da casca, resultado indesejável para comercialização destes frutos, apresentado aos 3 dias de armazenamento. Chen et al. (2001) relatam que a perda de massa superior a $18,21 \%$ é suficiente para causar escurecimento total do pericarpo de lichias, enquanto Bryant (2004) considera que uma perda de $3-5 \%$ pode causar este efeito.

Observa-se redução intensa na atividade respiratória, no segundo dia de armazenamento, a partir do qual se manteve praticamente inalterada, apresentando assim um padrão não climatérico (Figura 1B). Este comportamento também foi observado por Paull e Chen (1987) ao armazenarem lichias 'Chen Zi' a $22^{\circ} \mathrm{C}$.

Os frutos que receberam o tratamento hidrotérmico ou com $\mathrm{HCl}$ apresentaram, no $1^{\circ}$ dia, atividade respiratória mais intensa, $73,03 \mathrm{~mL}$ de $\mathrm{CO}_{2}$ $\mathrm{kg}^{-1} \mathrm{~h}^{-1}$ e 85,61 mL de $\mathrm{CO}_{2} \mathrm{~kg}^{-1} \mathrm{~h}^{-1}$, respectivamente, quando comparada com a dos frutos Testemunha,

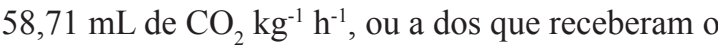

tratamento hidrotérmico seguido de resfriamento em $\mathrm{HCl}, 59,88 \mathrm{~mL}$ de $\mathrm{CO}_{2} \mathrm{~kg}^{-1} \mathrm{~h}^{-1}$. Isto, provavelmente, foi devido ao estresse sofrido pelos frutos na colheita e tratamento.

Observou-se, em todos os tratamentos, que o teor de sólidos solúveis (SS) aumentou linearmente durante o período de armazenamento (Figura 1C) como efeito da perda de massa, enquanto o de acidez titulável (AT) diminuiu (Figura 1D). Jiang et al. (2004) também relataram diminuição nos valores de acidez titulável, de $0,61 \%$ a $0,58 \%$, em lichias tratadas com $\mathrm{HCl}$ a $1 \%$ e de $0,69 \%$ a $0,61 \%$ em frutos não tratados, após 12 dias de armazenamento. Esta redução indica que os ácidos orgânicos devem ter sido utilizados como substratos respiratórios e como esqueletos de carbono para a síntese de novos compostos (CHITARRA; CHITARRA, 2005). Comportamento semelhante também foi verificado em lichias 'Groff' por Holcroft e Mitcham (1996), em 'Brewster' por Rivera-López et al. (1999) e em 'Huaizhi' por Jiang et al. (2004).

Peng e Cheng (2001) também não observaram efeito significativo do tratamento hidrotérmico (98 ${ }^{\circ} \mathrm{C}$ ), por 3 segundos, seguido de resfriamento em ácido $(\mathrm{pH}$ 0,5), por 5 minutos, no teor de sólidos solúveis de lichias 'Heye'.

Observou-se interação significativa entre os tratamentos e o tempo de armazenamento na relação entre os teores de sólidos solúveis e de acidez titulável (SS/AT), que aumentou durante o período de armazenamento (Figura 1E).

Os teores de ácido ascórbico na polpa dos frutos tratados com $\mathrm{HCl}$ a $1 \%$, por 6 minutos, apresentaram-se menores que nos frutos submetidos aos outros tratamentos (Figura 1F), o que pode ser resultado de oxidações dos ácidos orgânicos durante o armazenamento, e não foi observado por Jiang et al. (2004) em lichias 'Huaizhi'. Foyer et al. (1994) explicaram que, durante a senescência, o ácido ascórbico do fruto é utilizado em reações oxidativas, que são ativadas pelos estresses sofridos pelas membranas celulares durante este período.

A avaliação subjetiva da aparência indica que, nos frutos tratados com $\mathrm{HCl}$, a conservação da aparência foi melhor, principalmente naqueles frutos que foram resfriados em $\mathrm{HCl}$ a $1 \%$, após o tratamento hidrotérmico (Hidro $+\mathrm{HCl}$ ), em que a manutenção da nota 4 ou até $25 \%$ de escurecimento foi de 2 dias, e a nota 3 ou $50 \%$ de escurecimento foi de até 4 dias (Figura 2A). Este efeito também foi relatado por Huang e Scott (1985), que trabalharam com lichias 'Sui Dong', armazenadas a $28-31{ }^{\circ} \mathrm{C}$ e $90-95 \%$ de UR. Souza et al. (2010), estudando lichias 'Bengal' submetidas a tratamento hidrotérmico, concluíram 
que os melhores tratamentos para a manutenção da aparência dos frutos foram os de 5 e 10 minutos de imersão em água a $45^{\circ} \mathrm{C}$. Os tratamentos que foram submetidos por $15 ; 20$ e 25 minutos apresentaram diminuição significativa nos valores deste parâmetro.

Lichter et al. (2000), no entanto, ao armazenarem lichias 'Mauritius' por 3 semanas, a $1,5^{\circ} \mathrm{C}$ e 3 dias a $20^{\circ} \mathrm{C}$, após pulverização com água quente $\left(55^{\circ} \mathrm{C}\right.$ por 20 segundos), seguido de tratamento com ácido clorídrico ( $4 \%$ por $15-30$ minutos), relataram que a cor vermelha manteve-se por até 35 dias, mas sem que a ocorrência de manchas marrons e rachaduras na casca, que não comprometia a qualidade interna ou o sabor, fosse considerada.

Os resultados apresentados na Figura 2B indicam que houve redução significativa no teor de antocianina da casca dos frutos, submetidos aos diferentes tratamentos, durante o armazenamento. A melhor conservação deste pigmento foi naqueles frutos que receberam o tratamento hidrotérmico, com resfriamento em $\mathrm{HCl}$, seguido dos tratados com $\mathrm{HCl}$. A manutenção dos teores mais elevados de antocianinas na casca é resultado de sua degradação menos intensa e tem relação direta com a preservação da aparência, pois o escurecimento tem sido atribuído à degradação deste pigmento pela ação de enzimas oxidativas, polifenoloxidase, peroxidase e ácido ascórbico oxidase (UNDERHILL, 1992). No entanto, Lee e Wicker (1991) não observaram relação entre os teores de antocianinas e a cor vermelha do pericarpo de lichias.

Em lichias 'Wai Chee' tratadas com $\mathrm{HCl}$ a $1 \mathrm{M}$, por 2 minutos, e armazenadas a $25^{\circ} \mathrm{C}$ e $60 \% \mathrm{UR}$, Underhill e Critchley (1994) também observaram redução no teor de antocianina, sem que houvesse relação entre esta taxa de redução e a perda de massa. Quando armazenaram lichias 'Bengal' a $25^{\circ} \mathrm{C}$ e $60 \%$ UR estes autores também relataram decréscimo no teor de antocianina, mas relacionaram esta degradação com a perda de umidade e o desenvolvimento de escurecimento no pericarpo (UNDERHILL; CRITCHLEY, 1993), o que foi reafirmado por Zhang et al. (2001), ao armazenarem lichias 'Huaizhi' a $30^{\circ} \mathrm{C}$ e $70 \%$ UR e também foi o observado neste trabalho (Figuras 1A e 2B).

Os resultados obtidos neste trabalho parecem indicar que a atividade da polifenoloxidase (PPO) na casca das lichias submetidas aos diferentes tratamentos e armazenadas a $20{ }^{\circ} \mathrm{C}$ e $82 \%$ UR aumentou em todos os tratamentos até o $3^{\circ}-4^{\circ}$ dias, seguidos de estabilização (Figura 2C). A atividade desta enzima foi maior nos frutos Testemunha, com aumento de 6,31 para 10,88 $\mu$ mol de fenol consumido $\min ^{-1} \mathrm{~g}^{-1}$, enquanto no tratamento com imersão em $\mathrm{HCl}$ aumentou de 4,50 para 7,88 $\mu \mathrm{mol}$ de fenol consumido min $^{-1} \mathrm{~g}^{-1}$, no Hidrotérmico, de 4,08 para 8,25 $\mu \mathrm{mol}$ de fenol consumido $\min ^{-1} \mathrm{~g}^{-1}$, e no Hidrotérmico seguido de resfriamento em $\mathrm{HCl}$, de 3,55 para 7,45 $\mu$ mol de fenol consumido $\min ^{-1} \mathrm{~g}^{-1}$. Aumento semelhante, durante os primeiros dois dias de armazenamento, também foi relatado por Lin et al. (1988), enquanto Underhill e Critchley (1993 e 1994), Lichter et al. (2000) e Souza et al. (2010) relataram redução progressiva nesta atividade. Zauberman et al. (1991) não encontraram mudanças significativas na atividade da PPO em lichias 'Mauritius' durante este período. Estes resultados discordantes podem ser devidos a diferenças entre as metodologias utilizadas e/ou cultivares estudadas.

A atividade da peroxidase (POD) na casca dos frutos do tratamento-testemunha foi reduzida, enquanto nos tratados a POD aumentou até o $6^{\circ}$ dia (Figura 2D). Nos tratados hidrotermicamente, resfriados ou não em $\mathrm{HCl}$, este aumento foi bastante reduzido, apesar da possibilidade de regeneração parcial da atividade da peroxidase após o tratamento hidrotérmico (KHAN; ROBINSON, 1993).

Observou-se, também, que a atividade da polifenoloxidase e da peroxidase na casca dos frutos submetidos ao tratamento hidrotérmico, seguido de resfriamento em $\mathrm{HCl}$ a $1 \%$ (Hidro $+\mathrm{HCl}$ ), foi a mais reduzida, indicando que o tratamento utilizado impediu a degradação da antocianina, o que vem ao encontro dos teores de antocianina e das notas obtidas para a aparência. Outros autores também observaram que a inibição na atividade da PPO e da POD retardou o escurecimento do pericarpo em lichias (JIANG; FU, 1999; JIANG et al., 2004; SOUZA et al., 2009; SAAVEDRA DELL AGUILLA et al., 2009) e que há correlação positiva entre a atividade da POD (UNDERHILL ; CRITCHLEY, 1994) e da PPO (JIANG, 2000) com o escurecimento celular.

Segundo Mizobutsi et al. (2010), a cor vermelha da casca de lichias pode ser preservada se reduzir a atividade da peroxidase e da polifenoloxidase, o que pode ser conseguido com a imersão dos frutos em soluções ácidas ou alcalinas. Resultados semelhante foram encontrados por Silva et al. (2010) em lichias 'Bengal' armazenadas a $5{ }^{\circ} \mathrm{C} \pm 1,2{ }^{\circ} \mathrm{C}$ e $90 \%$ UR por 12 dias, tratadas com $15 \mathrm{Mm}$ e $30 \mathrm{mM}$ de ácido ascórbico; entretanto, observou-se escurecimento a partir do quarto dia no pericarpo dos frutos tratados com este ácido.

Não se detectou atividade da polifenoloxidase ou da peroxidase na polpa dos frutos submetidos aos diferentes tratamentos, sugerindo que o escurecimento está restrito ao pericarpo do fruto, o que reafirma o relatado por Underhill e Critchley (1993). 


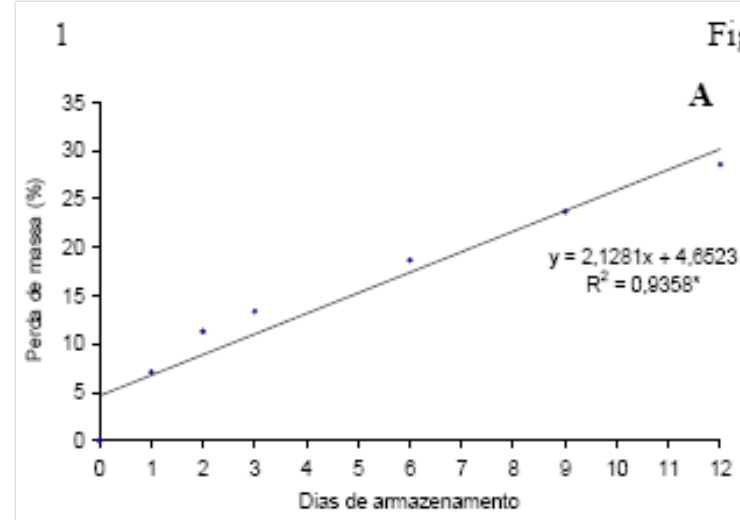

Figuras
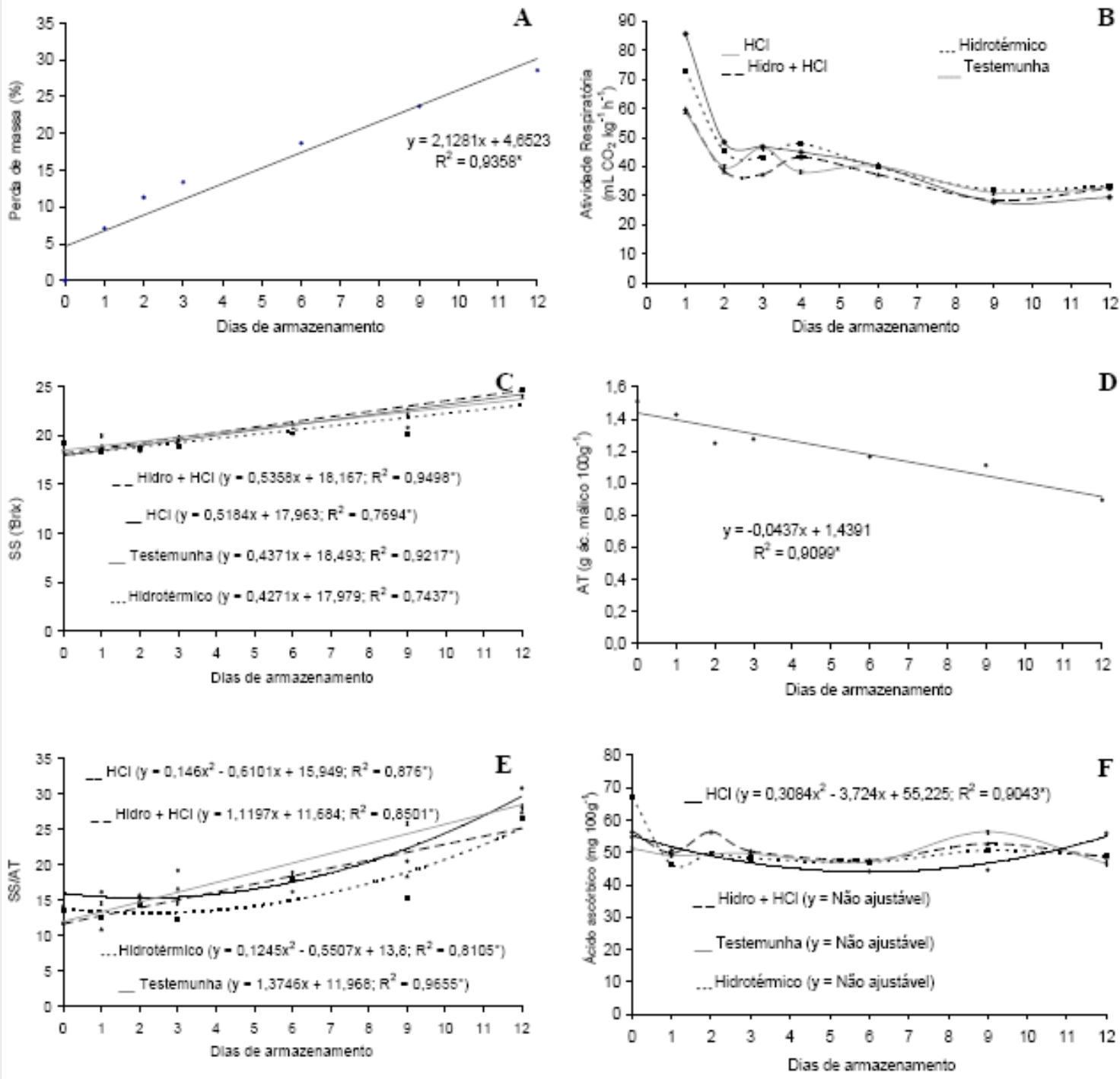

FIGURA 1 - Perda de massa (A), atividade respiratória (B), teores de sólidos solúveis - SS (C), acidez titulável - AT (D), SS/AT (E) e ácido ascórbico (F) da polpa em lichias 'Bengal' submetidas a tratamento hidrotérmico, associado ou não com imersão em $\mathrm{HCl}$ a $1 \%$, e armazenadas a $20{ }^{\circ} \mathrm{C}$ e $82 \% \mathrm{UR}$. 

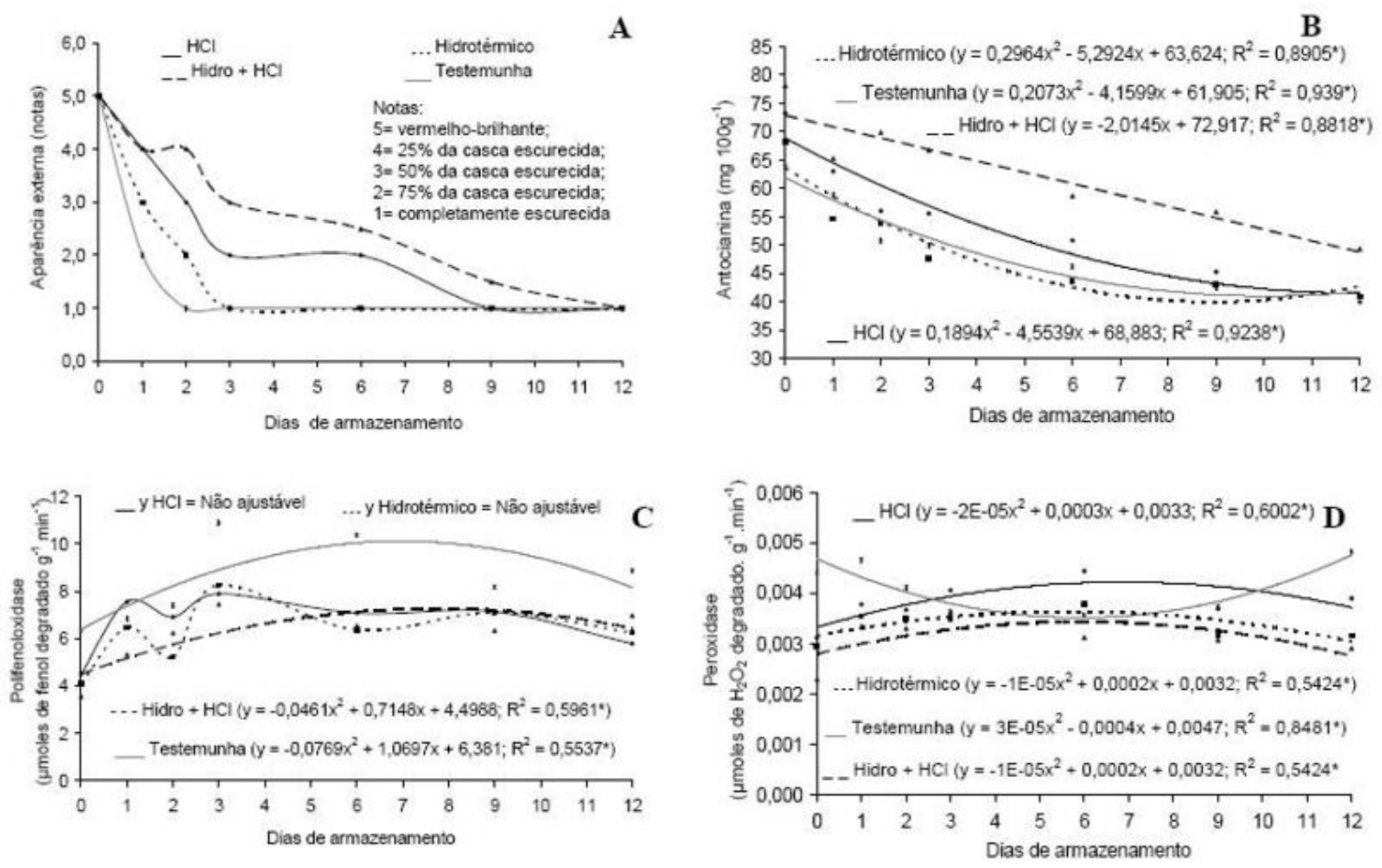

FIGURA 2 - Aparência dos frutos (A), teor de antocianina (B), atividade da polifenoloxidase (C) e da peroxidase (D) na casca de lichias 'Bengal' submetidas a tratamento hidrotérmico, associado ou não com imersão em $\mathrm{HCl}$ a $1 \%$, e armazenadas a $20^{\circ} \mathrm{C}$ e $82 \% \mathrm{UR}$.

\section{CONCLUSÕES}

A combinação entre tratamento hidrotérmico $\left(52{ }^{\circ} \mathrm{C}\right.$ por 1 minuto) e resfriamento em $\mathrm{HCl}$ a $1 \%$ permite conservar a coloração das lichias 'Bengal' por até dois dias. Mesmo assim, há escurecimento em $25 \%$ da superfície.

\section{AGRADECIMENTOS}

À FAPESP (Processo 07/57351-9) e ao $\mathrm{CNPq}$, pela concessão de bolsa de doutorado.

\section{REFERÊNCIAS}

ALlAin, C. C.; POON, L. S.; CHAN, C. S. G.; RICHMOND, W.; FU, P.C. Enzymatic determination of total serum colesterol. Clinical Chemistry, Baltimore, v. 120, p. 470-475, 1974.

A.O.A.C. Official methods of analysis. Arligton: Patrícia Cuniff, 1997. p.37-10, 42-2, 44-3, 45-16.
BRYANT, P. Optimising the postharvest management of lychee (Litchi chinensis Sonn.) - a study of mechanical injury and desiccation. 2004. 397 f. Thesis (Doctor of Philosophy) - Department of Crop Sciences, University of Sydney, Sydney, 2004.

CHEN, W.; WU, Z.; JI, Z.; SU, M. Postharvest research and handling of litchi in China - a review. Acta Horticulturae, Netherlands, n. 558, p. 321$329,2001$.

CHITARRA, M. I. F.; CHITARRA, A. B. Pós-colheita de frutos e hortaliças: fisiologia e manuseio. 2.ed. Lavras: Ed. UFLA, 2005. 783 p.

FOYER, C. H.; DESCOURVIERES, P.; KUNERT, K. J. Protection against oxygen radicals: An important defense mechanism studied in transgenic plants. Plant Cell and Environment, Malden, v. 17, p. 507-523, 1994.

FRANCIS, F.J. Analysis of anthocyanins. In: MARKAKIS, P. (ed.). Anthocyanins as food colors. New York: Academic Press, 1982. p.181-207. 
HOLCROFT, D. M.; MITCHAM, E. J. Review: postharvest physiology and handling of litchi (Litchi chinensis Sonn.). Postharvest Biology and Technology, Amsterdam, v.9, n.1, p.265-281, 1996.

HUANG, P. Y.; SCOTT, K. J. Control of rotting and browning of litchi fruit after harvest at ambient temperatures in China. Tropical Agriculture, Trinidad, n. 1, v. 62, p. $1-4,1985$.

JIANG, Y.; FU, J. Inhibition of polyphenol oxidase and browning control of litchi fruit by glutathione and citric acid. Food Chemistry, Oxford, v.62, n.1, p.49-52, 1999.

JIANG, Y. M. Role of anthocyanins, polyphenol oxidase and phenol in lychee pericarp browning. Journal of Science of Food and Agriculture, Hoboken, v. 80, p. 305-310, 2000.

JIANG, Y.; LI, Y.; LI, J. Browning control, shelf life extension and quality maintenance of frozen litchi fruit by hydrochloric acid. Journal of Food Engineering, Essex, v. 63, p.147-151, 2004.

KHAN,A.A.; ROBINSON, D. S. The thermostability of purified mango isoperoxidases. Food Chemistry, Oxford, v. 47, n. 4, p. 53-59, 1993.

LEE, H. S.; WICKER, L. Anthocyanin pigments in the skin of lychee fruit. Journal of Food Science, Chicago, v.56, p.466-468, 1991.

LICHTER, A.; DVIR, O.; ROT, I.; AKERMAN, M.; REGEV, R.; WIESBLUM, A.; FALLIK, E.; ZAUBERMAN, G.; FUCHS, Y. Hot water brushing: an alternative method to $\mathrm{SO}_{2}$ fumigation for color retention of litchi fruits. Postharvest Biology and Technology, Amsterdam, v. 18, p.235-244, 2000.

LIN, Z.; LI, S. S.; CHANG, D. L.; LIN, G. Z.; LI, Y. B.; LIU, S. X.; CHEN, M. D. The changes of pigments, phenolic content and activities of polyphenol oxidase and phenylalanine amoonia-lyase in pericarp of postharvest litchi fruit. Acta Botanica Sinica, Beijing, v.30, n.1, p.40-45, 1988.

MIZOBUTSI, G. P.; FINGER, F. L.; RIBEIRO, R. A.; PUSCHMANN, R.; NEVES, L. L. de M ; MOTA, W. F. Effect of $\mathrm{pH}$ and temperature on peroxidase and polyphenoloxidase activities of litchi pericarp. Scientia Agrícola, Piracicaba, v.67, n.2, p.213-217, 2010.
PAULL, R. E.; CHEN, N. J. Effect of storage temperature and wrapping on quality characteristics of litchi fruit. Scientia Horticulturae, Netherlands, v. 33, n. 3-4, p. 223-236, 1987.

PENG, Y. H.; CHENG, W. Effect of postharvest handling on fruit quality, mass loss and respiration rate of litchi. Acta Horticulturae, Amsterdam, n.558, p.359-365, 2001.

RANGANNA, S. Manual of analysis of fruit and vegetable products. New Delhi: McGraw-Hill, 1977. 634p.

RIVERA-LOPEZ, J.; ORDORICA-FALOMIR, C.; WESCHE-EBELING, P. Changes in anthocyanin concentration in Lychee (Litchi chinensis Sonn.) pericarp during maturation. Food Chemistry, Oxford, v.65, p.195-200, 1999.

SAAVEDRA DEL AGUILA, J.; DEL AGUILA, H., L. S.; SASAKI, F. F. ; ORTEGA, E. M. M.; KLUGE, R. A. Efeito de antioxidante na taxa respiratória e na produção de etileno de lichia 'Bengal' armazenada sob refrigeração. Revista Ibericoamericana de Tecnologia Postcosecha, Hermosillo, v. 10, n. 1, p. 8-13, 2009.

SIVAKUMAR, D.; KORSTEN, L. Influence of modified atmosphere packaging and postharvest treatments on quality retention of litchi $\mathrm{cv}$. Mauritius. Postharvest Biology and Technology, Amsterdam v. 41, p.135-142, 2006.

SILVA, D. F. P. DA; CABRINI, E. C.; ALVES, R. R.; SALOMÃO, L. C. C. Uso do ácido ascórbico no controle do escurecimento do pericarpo de lichia. Revista Brasileira de Fruticultura, Jaboticabal, v. 32 , n. 2, p. 1-7, 2010.

SOUZA, A. V. DE; VIEITES, R. L.; KOHATSU, D. S.; LIMA, G. P. P. Manutenção da coloração da lichia frigorificada com a utilização de ácidos orgânicos. Revista Ibericoamericana de Tecnologia Postcosecha, Hermosillo, v. 10, n. 1, p. 67-73, 2010.

SOUZA, A. V. DE; VIEITES, R. L.; KOHATSU, D. S.; LIMA, G. P. P. Tratamento térmico na manutenção da coloração de lichias. Revista Brasileira de Fruticultura, Jaboticabal, v. 32, n. 1, p. 67-73, 2010.

UNDERHILL, S. J. R. Lychee (Litchi chinensis Sonn.) pericarp browning. Tropical Science, London, v.32, n.3, p.305-312, 1992. 
UNDERHILL, S.J.R.; COATES, L.M.; SAKS, Y. LITCHI. In: MITRA, S.K. (Ed.). Postharvest physiology and storage of tropical and subtropical fruits. London: CAB International, p.191-208, 1997.

UNDERHILL, S. J. R.; CRITCHLEY, C. Anthocyanin decoloration and its role in lychee pericarp browning. Australian Journal of Experimental Agriculture, Melbourne, v.34, n.1, p.115-122, 1994.

UNDERHILL, S.J.R.; CRITCHLEY, C. Physiological, biochemical and anatomical changes in lychee (Litchi chinensis Sonn.) pericarp during storage. Journal of Horticultural Science, Ashford, v.68, n.3, p.327-335, 1993.
ZAUBERMAN, Q.; RONEN, R.; AKERMAN, M.; WESLER, A.; ROT, I.; FUCHS, Y. Postharvest retention of the red colour of litchi fruit pericarp. Scientia Horticulturae, Amsterdam, v.47, n.1, p.89-97, 1991.

ZHANG, Z.; PANG, X.; JI, Z.; JIANG, Y. Role of anthocyanin degradation in litchi pericarp browning. Food Chemistry, Oxford, v. 75, p. 217-221, 2001. 